\title{
Clinical Case: Dexmedetomidine for Non-invasive Mechanical Ventilation in Postoperative Cardiac Surgery
}

Baena López M, Raigón Ponferrada A, Guerrero Orriach Jose L, Ramirez Fernandez A, Ramirez Aliaga M, Loras P, Gallego M, Bermudez L, Biteri A, Rodriguez Capitan MJ, Rubio Navarro M and Cruz Mañas J

Department of Anaesthesia, Hospital Virgen de la Victoria, Málaga, Spain

${ }^{*}$ Corresponding author: Jose GOL, Department of Anaesthesia, Hospital Virgen de la Victoria, Málaga, Spain, Tel: 0034951032217; E-mail: guerreroorriach@gmail.com

Rec date: May 17, 2016; Acc date: May 27, 2016; Pub date: May 31, 2016

Copyright: ( 2016 López GoL, et al. This is an open-access article distributed under the terms of the Creative Commons Attribution License, which permits unrestricted use, distribution, and reproduction in any medium, provided the original author and source are credited.

\begin{abstract}
Dexmedetomidine is a new drug often used in the treatment of neurological disorders in intensive care unit thanks to its effects $\alpha 2$. In our case we needed to use dexmedetomidine in a patient with a heart failure after cardiac surgery in which respiratory function was supported with noninvasive mechanical ventilation. Dexmedetomidine was given successfully after has tried the treatment with opioids, opening the door as alternative treatment in patients with heart failure and noninvasive mechanical ventilation with $\alpha 2$ agents.
\end{abstract}

Keywords: Noninvasive; Mechanical ventilation; Dexmedetomidine; Cardiac surgery; Neurological disorder

\section{Introduction}

Noninvasive mechanical ventilation (NIMV) is a method of mechanical ventilation that has great benefits in certain patient groups. Those include patients with decompensated respiratory disease or who are in situations of heart failure and respiratory failure. Versus invasive mechanical ventilation, there is a decrease of mortality at some patients groups, including into them those diagnosed as acute pulmonary edema. This is due to decreased complications and benefits at respiratory and haemodynamic level, by reducing venous return and facilitating left ventricular ejection as a result of the transmitted positive pressure [1].

\section{Case Report}

For the use of NIMV it is essential a proper patient sedation so that his anxiety decreases while increasing his synchrony with the fan. One of the main reasons for failure of this therapy is the lack of collaboration or the intolerance of it.

The most commonly used drugs to promote tolerance of NIMV are propofol, benzodiazepines and opiates. In some patients the side effects of these drugs prevent the use of this technique (impaired level of consciousness, cardiocirculatory depression, respiratory depression stimulus, or agitation). Currently available drugs are able to provide sedation, hypnosis, analgesia with minimal risk of respiratory depression, reducing the need for high doses of morphine and enhace the effects of these drugs [2].

Dexmedetomidine, through its agonist effect on a2-adrenergic receptors, produces a sedative and analgesic effect without associated respiratory depression, with broad therapeutic range. Furthermore it is also associated a lower incidence of delirium [3]. It is not recommended to administer an initial loading dose due to the appearance of cardiovascular instability, tachycardia, bradycardia, or heart block. The usual dexmedetomidine maintenance dose is 0.2 to
$0.7 \mathrm{mcg} / \mathrm{kg} / \mathrm{h}$, but it may also be gradually adjusted until the desired level of sedation according to patient response, up to $1.5 \mathrm{mcg} / \mathrm{kg} / \mathrm{h}$. Reported adverse effects include hypotension, hypertension, bradycardia, nausea and atrial fibrillation [4]. As monitoring parameters may include, heart rate, respiration, rhythm, blood pressure, pain control and depth of sedation with either the Richmond Agitation-Sedation Scale (RASS) or Sedation-Agitation Scale (SAS).

Patient 66 years old with a history of hypertension, chronic renal failure and chronic pulmonary respiratory disease; is diagnosed with severe aortic stenosis and coronary disease with involvement of two coronary arteries: anterior descending and circumflex; surgery is decided. At surgery it was performed aortic valve replacement and myocardial revascularization of both vessels. In the intensive care unit the patient has coronary ischemic event within 24 hours of arrival, in need of urgent myocardial revascularization after diagnosing coronary graft obstruction of the descending anterior. There was a hemodynamic deterioration that required support with adrenaline 0.07 $\mathrm{mcg} / \mathrm{kg} / \mathrm{min}$ and levosimendan $0.2 \mathrm{mcg} / \mathrm{kg} / \mathrm{min}$ continuous infusions.

Patient's respiratory status worsens progressively with a ratio of $\mathrm{PaO} 2 / \mathrm{FiO} 2$ of 130 , a respiratory rate of 35 breaths/min and dyspnea. It was decided to force diuresis with intravenous furosemide and perform treatment with NIMV; morphine hydrochloride was administered in two doses of 5 milligrams intravenous, and the NIMV started with values of $5 \mathrm{~cm} \mathrm{H} 2 \mathrm{O}$ PEEP and $5 \mathrm{~cm} \mathrm{H} 2 \mathrm{O}$ supports. After 30 minutes patient refered didn't tolerate NIMV, with a state of agitation ( +2 degree on the scale of Richmond (RASS) and $\mathrm{PaO} 2 / \mathrm{FiO} 2$ fell down again until 110. It was decided to use dexmedetomidine continuous infusion without bolus infusion at a rate between $0.3 \mathrm{mcg} /$ $\mathrm{kg} / \mathrm{h}$ and $0.5 \mathrm{mcg} / \mathrm{kg} / \mathrm{h}$, getting a sedation level between 0 and -1 of the RASS scale. The patient was collaborator and comfortable, and allowed the gradual withdrawal of adrenaline while maintaining stability during NIMV. After 12 hours NIMV was removed when the oxigenation had improved reaching $\mathrm{PaO} 2 / \mathrm{FiO} 2$ of 320 , and the infusion of dexmedetomidine was stopped after removed the NIMV. Patient was discharged after 96 hours. 
Citation: López BM, Ponferrada RA, Jose GOL, Fernandez RA, Aliaga RM, et al. (2016) Clinical Case: Dexmedetomidine for Non-invasive Mechanical Ventilation in Postoperative Cardiac Surgery. J Cardiovasc Dis Diagn S1: 009. doi:10.4172/2329-9517.1000S1-009

Page 2 of 2

The use of dexmedetomidine as a rescue technique in patients with acute pulmonary edema who do not cooperate after a first attempt sedation with another group of drugs is documented in some studies, as well as in patients with decompensated chronic respiratory disease. In our case we have a patient with respiratory disease and cardiac failure, in one of the most serious complications in the postoperative period of myocardial revascularization: acute myocardial infarction after surgery.

The need for sedation of comfort in acute pulmonary edema made us use initially morphine chloride in order to get a double benefit in the treatment of dyspnea and adapting to the NIMV, which however failed. Subsequently, in the state of agitation of the patient we decided to start the infusion of dexmedetomidine, now successfully. In the scientific literature may be found sedation with dexmedetomidine experiences with great success of the technique, reaching the conclusion that it is able to reduce respiratory complications associated with NIMV and even the worsening of respiratory mechanics with subsequent intubation. In our case was necessary a high control of the sedation level with a sedation scale (in our case Richmond scale), because the interaction between both drugs could have produced more adverse effects as apnea, hypotension or bradycardia [5].

There are many drugs used trying to improve collaboration and comfort of patients who used the NIMV. Dexmedetomidine compared to midazolam reduces ICU stay, duration of mechanical ventilation, the need for endotracheal intubation and hospital-acquired infections [6]. In addition to situations of NIMV failure after administration of other sedative drugs, other reports suggest the early use of dexdemetomidine in patients when noninvasive ventilation is decided as a treatment to acute respiratory failure [7].

To conclude we could say that randomized studies are needed to confirm the possibility of using dexmedetomidine as first choice drug in patients at high risk of respiratory failure, such as the patient we are talking about, in which lung disease was associated with acute pulmonary edema, which require NIMV.

\section{References}

1. Masip J, Roque M, Sánchez B, Fernández R, Subirana M, et al. (2005) Noninvasive ventilation in acute cardiogenic pulmonary edema: systematic review and meta-analysis. JAMA 294: 3124-3130.

2. Afonso J, Reis F (2012) Dexmedetomidine: current role in anesthesia and intensive care. Rev Bras Anestesiol 62: 118-133.

3. Ortega RMA, Jambrina CCI, Vallhonrat LI, Simón IF (2014) Indications of dexmedetomidine in the current sedoanalgesia tendencies in critical patients. Med Intensiva 38: 41-48.

4. Tietze KJ, Fuchs B (2016) Sedative-analgesic medications in critically ill adults: Properties, dosage regimens, and adverse effects.

5. Demuro JP, Mongelli MN, Hanna AF (2013) Use of dexmedetomidine to facilitate non-invasive ventilation. Int J Crit Illn Inj Sci 3: 274-275.

6. Huang Z, Chen YS, Yang ZL, Liu JY (2012) Dexmedetomidine versus midazolam for the sedation of patients with non-invasive ventilation failure. Intern Med 51: 2299-2305.

7. Devlin JW, Al-Qadheeb, Chi A, Roberts RJ, Qawi I, et al. (2014) Efficacy and safety of early dexmedetomidina during noninvasive ventilation for patients with acute respiratory failure. CHEST 145: 1204-1212. 